DOI: $10.15593 / 2224-9982 / 2020.63 .03$

УДК 623.743

А.А. Дидковский, Е.Д. Мухина, А.К. Чернов

Московский государственный технический университет им. Н.Э. Баумана, Москва, Россия

\author{
РАЗРАБОТКА ТРАНСПОРТНОГО ДИРИЖАБЛЯ ДЛЯ ДОСТАВКИ \\ НЕГАБАРИТНЫХ ГРУЗОВ НА КОСМОДРОМ «ВОСТОЧНЫЙ»
}

\begin{abstract}
Проведен обзор существующих способов доставки негабаритных грузов при помощи водного, железнодорожного, автомобильного, авиационного и дирижабельного транспорта. Проведен анализ мирового опыта создания летательных аппаратов легче воздуха и выявлены наиболее актуальные задачи для аппаратов данного типа в области ракетнокосмической техники, в частности транспортировка негабаритных элементов сверхтяжелых ракет-носителей с заводовизготовителей на космодром «Восточный», поиск и спасение космонавтов, эвакуация отработавших ступеней с полей падения к точкам утилизации и перевозка негабаритных ферменных конструкций. Определены цели и задачи настоящей работы: обзор существующих проектов транспортных дирижаблей и изучение их конструкций, разработка компоновки транспортного дирижабля для доставки ступеней сверхтяжелых ракет-носителей с заводов-изготовителей на космодром «Восточный», расчет характеристик основных систем и детализация конструкции транспортного средства. В качестве исключительных конструкторских решений опробованы силовая схема газовых оболочек на основе наддуваемых спиральных пневмоконструкций, которые позволяют термостатировать несущий газ и складывать оболочку для хранения, а также тангенциальная система обдува аэродинамической оболочки, позволяющая снизить лобовое сопротивление аппарата. На основе разработанной компоновки была создана упрощенная математическая модель аппарата, позволяющая определить оптимальные проектные параметры. Данные параметры были использованы при детальной проработке каждой из систем, затем производился итерационный расчет. В результате работы разработан облик транспортного дирижабля для доставки негабаритных элементов с заводов-изготовителей на космодром «Восточный» и определены его основные технические параметры: грузоподъемность - 100 т, крейсерская скорость - 126 км/ч, дальность полета - 10 тыс. км.

Ключевые слова: транспорт, воздухоплавание, аппараты легче воздуха, дирижабль, грузоперевозки, транспортировка элементов РКТ, ракетная техника, сверхтяжелый класс, негабаритные грузы, космодром «Восточный», спасение космонавтов, утилизация отработавших ступеней.
\end{abstract}

\author{
A.A. Didkovskiy, E.D. Mukhina, A.K. Chernov \\ Moscow State Technical University named after N.E. Bauman, \\ Moscow, Russian Federation
}

\title{
DEVELOPING OF TRANSPORT AIRSHIP FOR DELIVERY OVERSIZED CARGO TO VOSTOCHNY COSMODROME
}

An overview of existing methods of delivering oversized cargo using water, rail, road, air and airship transport, an analysis of the world experience in creating lighter-than-air aircraft and identified the most urgent tasks for this type of vehicles in the field of rocket and space technology, in particular, transportation of oversized elements of super-heavy launch vehicles from manufacturers to the Vostochny cosmodrome, search and rescue of astronauts, evacuation of spent stages from fall fields to disposal points and transportation of oversized trusses. The goals and objectives of this work are defined: review of existing projects of transport airships and study of their designs, development of the layout of a transport airship for delivering stages of superheavy launch vehicles from manufacturers to the Vostochny cosmodrome, calculation of the characteristics of the main systems and detailing the design of the vehicle. As exceptional design solutions, we tested the power scheme of gas shells based on supercharged spiral pneumatic structures that allow you to thermostat the carrier gas and fold the shell for storage, as well as a tangential system for blowing the aerodynamic shell, which allows you to reduce the drag of the device. Based on the developed layout, a simplified mathematical model of the device was created, which allows determining the optimal design parameters. These parameters were used for detailed development of each of the systems, then iterative calculation was performed. As a result, the design of a transport airship for the delivery of oversized elements from manufacturers to the Vostochny cosmodrome was developed and its main technical parameters were determined: load capacity-100 tons, cruising speed-126 $\mathrm{km} / \mathrm{h}$, flight range-10 thousand $\mathrm{km}$.

Keywords: transport, aeronautics, lighter-than-air vehicles, airship, cargo transportation, transportation of RCT elements, rocket technology, super-heavy class, oversized cargo, Vostochny cosmodrome, rescue of cosmonauts, disposal of spent stages. 


\section{Введение}

Как правило, космодромы и предприятия производители ракетно-космической техники находятся друг от друга на значительном расстоянии, в связи с чем возникает проблема транспортировки соответствующих изделий (блоков ракет-носителей $(\mathrm{PH})$, космических аппаратов (КА), элементов наземной инфраструктуры и пр.). Грузы ракетно-космической техники имеют ряд особенностей, которые усложняют их доставку. К ним относятся: негабаритность, большая масса, высокие требования по отсутствию перегрузок, вибраций и ударов.

К наиболее распространенным существующим видам транспорта, осуществляющим доставку таких грузов, относятся: железнодорожный транспорт, автотранспорт, водный транспорт и перевозка самолетом.

Каждый из приведенных выше способов доставки грузов ракетно-космической техники имеет свои преимущества и недостатки и является оптимальным лишь для определенных требований перевозок. Играют роль дальность и время транспортировки, наличие объектов транспортной инфраструктуры (железная дорога, автомобильная дорога, водные пути, взлетно-посадочные полосы и пр.), форма, габарит и массоинерционные характеристики груза, требования по перегрузкам, вибрации, температурному режиму и пр.

В связи с разработкой лунной программы [1] в 2020-2040 гг. возникнет необходимость транспортировки блоков тяжелых и сверхтяжелых ракет с заводов-изготовителей на космодром «Восточный». Относительно низкая частота запусков и специфика каждого груза делают экономически невыгодным разработку и использование самолетов, габариты грузов и отсутствие у предприятий - производителей ракетно-космической техники прямого выхода к морю не позволят широко использовать железнодорожный и водный транспорт [2-4].

Таким образом, в отрасли в настоящий момент существует проблема доставки негабаритных грузов ракетно-космической техники (РКТ) (блоки РН сверхтяжелого класса (СТК), КА, негабаритные элементы наземной инфраструктуры). В качестве решения описанной проблемы предлагается применить транспортный дирижабль.
Цель работы - определение проектных параметров и разработка конструкции основных систем транспортного дирижабля для доставки негабаритных грузов РКТ с заводовизготовителей на космодром «Восточный».

Задачи работы:

1. Произвести анализ существующих проектов транспортных дирижаблей и сформировать комплекс ключевых конструкторских решений, определяющих облик дирижабля (тип силовой конструкции, аэродинамическая схема, несущий газ, тип движителя, система швартовки и пр.).

2. Рассчитать основные проектные параметры дирижабля (объем газовых оболочек, масса изделия, мощность энергетической установки, высота полета, крейсерская скорость и пр.).

3. На основе проектных параметров произвести расчет характеристик основных систем (силовая конструкция, движитель и элементы управления, система термостатирования, система швартовки и пр.).

4. Проработать конструкцию основных систем.

5. Произвести корректировочный расчет на основе уточненных характеристик основных систем.

6. Оценить экономические характеристики изделия как транспортного средства.

\section{Расчетная модель}

Основной задачей транспортной системы является доставка с заводов-изготовителей составных частей и ступеней ракет космического назначения на космодром: 1-я ступень РН СТК (2 ед.); 3-я ступень РН СТК (1 ед.), составляющие вместе с транспортным агрегатом массу порядка 90 т. К задачам также относятся: транспортирование крупногабаритных конструкций и элементов наземных комплексов; проведение поиска и эвакуации из районов падения отделяемых частей $\mathrm{PH}$; проведение поисково-спасательных и эвакуационных работ при возвращении КА; размещение на борту дирижабля радиолокационного оборудования.

Проведение подробного анализа мирового опыта создания подобных аппаратов [5-15] и анализ доставляемого груза позволили сформировать основную компоновку и технические характеристики дирижабля. Целевая вы- 
сота продолжительного полета составляет 6-8 км, крейсерская скорость 100-150 км/ч, размеры грузового отсека $11,5 \times 12 \times 55,4 \mathrm{M}$, масса полезной нагрузки 100 т.

Исходными данными для всех расчетов являются массогабаритные характеристики дирижабля. При этом точные значения массы отдельных систем и их характеристики на момент проектного расчета неизвестны.

Эксплуатационные условия были определены исходя из анализа данных радиозондирования атмосферы на высоте 0-8 км по маршруту Москва - Самара - космодром «Восточный».

На основе требуемой скорости выхода на крейсерский режим мы сформулировали выражение для оценки мощности двигательной установки:

$$
P_{\text {д }}=\frac{4\left(3 M_{\Gamma \Pi}\right)}{\eta_{\text {ад }}} \frac{v_{\text {к }}}{t_{V}} .
$$

На основе модели оболочки, отражающей форму дирижабля, были получены зависимости для площадей несущей фермы (НФ) и функциональной оболочки (ФО):

$$
\begin{aligned}
& S_{\mathrm{H} \Phi}(V)=1,29 V^{\frac{2}{3}} ; \\
& S_{\Phi О}(V)=6,46 V^{\frac{2}{3}} .
\end{aligned}
$$

Определено выражение для массы топлива на борту, а также массы самого аппарата:

$$
\begin{gathered}
M_{T}\left(V, v_{\mathrm{\kappa}}, h\right)=\frac{L_{\text {дал }}}{v_{\mathrm{\kappa}}}\left(C_{x} \frac{\rho_{\text {аіг }} v_{\mathrm{\kappa}}^{2}}{2} V^{\frac{2}{3}}\right) ; \\
M\left(V, v_{\mathrm{\kappa}}, h\right)=M_{\Gamma \Pi}+P_{\text {д } \rho_{\text {эн.уст }}+}+ \\
+M_{\mathrm{T}}\left(V, v_{\mathrm{\kappa}}, h\right)+S_{\mathrm{HФ}} \rho_{\mathrm{HФ}}+2 S_{\Phi О} \rho_{\Phi О}+\rho_{\text {air }} V .
\end{gathered}
$$

Нами была также решена задача оптимальной высоты и крейсерской скорости. С учетом того, что максимальная высота полета дирижабля увеличивается при увеличении подъемной силы, максимум высоты будет соответствовать максимуму скорости. Записав уравнения движения дирижабля, мы получим оптимальную высоту и крейсерскую скорость:

$$
\left\{\begin{array}{c}
M\left(V, v_{\mathrm{\kappa}}, h\right) g=C_{y} \frac{\rho_{\mathrm{air}} v^{2}}{2} V^{\frac{2}{3}}+\rho_{\mathrm{air}} g V ; \\
v C_{x} \frac{\rho_{\mathrm{air}} v^{2}}{2} V^{\frac{2}{3}}=P_{\text {д }} .
\end{array}\right.
$$

В качестве аэродинамической схемы принята гибридная схема. Для создания тяги используется технология тангенциального обдува оболочки. Воздух на всей площади выходит изпод оболочки по касательной в противоположном скорости дирижабля направлении. Такая система позволяет снизить лобовое сопротивление и расход топлива. Управление по рысканью осуществляется за счет разности расходов через щели на левом и правом бортах дирижабля, по тангажу - за счет стабилизатора.

Исходные данные по параметрам обтекания мы определили, проведя расчет в программном комплексе (ПК) SolidWorks Flow Simulation.

Сначала были определены параметры оболочки: количество щелей $N$, толщина щелей $\delta$ и диаметр импеллера $D_{\text {им }}$. Дистанция срыва потока

$$
x_{\text {кр }}=\frac{10^{6} v_{\text {нп }}}{v_{\text {нп }}} .
$$

Количество щелей определяется зависимостью

$$
N=\frac{1}{2}\left(\frac{D_{\text {адо }}}{x_{\text {кр }}}\left(\pi-\varphi_{0}\right)-1\right) .
$$

Ширина щели определяется главным из трех факторов: скоростью выхода на крейсерский режим (тягой), удвоенной толщиной ПС и минимальной шириной, которую можно обеспечить технологически:

$$
\begin{gathered}
\delta=\max \left(\frac{4 M v_{\text {ЛА }}}{\pi t_{v} \rho_{\text {возд }} D_{\text {адо }} v^{2} \sum_{i=\frac{\varphi_{0}}{\Delta \varphi} \mid+1}^{N} \sin ^{2}(i \Delta \varphi)},\right. \\
\left.50 \frac{v k}{v_{\text {нп }}}, \delta_{\text {технол }}\right) .
\end{gathered}
$$

Эквивалентный диаметр импеллера определяется исходя из расхода на всей группе щелей:

$$
D_{\text {им }}=2 \sqrt{\frac{D_{\text {адо }}}{v_{\text {им }}} \sum_{i=\left|\frac{\varphi_{0}}{\Delta \varphi}\right|+1}^{N} v_{\text {щi }} \delta_{i} \sin (i \Delta \varphi)} .
$$

Отсюда определяется площадь, соответствующая импеллеру. Эта площадь может быть образована группой из нескольких импеллеров. Необходимо также проверить реа- 
лизацию ламинарного режима течения в межоболочковом пространстве, что можно записать математически:

$$
\operatorname{Re}<\operatorname{Re}_{\text {кр }}
$$

Силовая схема представляет собой две продольных газовых оболочки, размещенных по пакетной схеме, укрепленных сверху несущей фермы, в центре которой размещен поперечный грузовой отсек. Оболочки выполнены по квазижесткой схеме. По внутренней поверхности оболочки размещены спиралевидные рукава, наддутые воздухом. Оболочка тросами стягивается к собственной оси. Тем самым достигается требуемая жесткость конструкции при минимальной массе. Наддутые рукава также являются хорошими теплоизоляторами. За счет системы откачки воздуха из оболочки и системы наружных тросов обеспечивается возможность автоматизированного сдува и наддува оболочки с целью снижения аэродинамических нагрузок во время стоянки. В процессе наддува спиралей с воздухом между спиралями возникают нормальные силы, которые стремятся расширить оболочку. При достижении заданного объема дальнейшему расширению препятствуют тросы, идущие из центра баллонета.

В качестве основы расчета нами был рассмотрен произвольный виток спирали и определены силовые факторы, возникающие в системе спиралей при нагрузке, приложенной к данному витку. Определена ключевая характеристика системы - жесткость. Она убывает до определенного значения перемещения тросов. Достижение минимума жесткости означает, что дальнейшее малое изменение площади контакта не оказывает влияние на жесткость. При дальнейшем проектировании первоначальное натяжение тросов необходимо выбрать больше минимального значения, чтобы жесткость конструкции при нагружении увеличивалась.

Далее нами было рассмотрено два расчетных случая: приложение нагрузки к центру газовой оболочки и приложение нагрузки при внешней распределенной нагрузке. Произведен оценочный расчет на прочность элементов спиралевидных рукавов. Произведена оценка влияния на жесткость применения двух слоев спиралей.
Для оценки теплоизоляционных свойств функциональной оболочки было также рассмотрено два варианта конструкции «стенки» газовой оболочки: с одним и двумя спиральными рукавами.

При разработке рассматриваемой конструкции оболочки дирижабля закладывалась «идея стеклопакета». В конструкции стенки газовой оболочки роль стеклопакета выполняет спиральный рукав с воздухом, поэтому при расчете тепловых потоков внутри спирального рукава используются методы, применяемые для вычисления тепловых потерь через ограждения с воздушными полостями.

Расчет проводился в программном комплексе Excel при помощи метода последовательных приближений.

Проверка теоретических расчетов методом конечных элементов (МКЭ) в ПК SolidWorks Simulation дала аналогичные результаты.

Для выбора оптимального варианта размеров и конструкции стенки газовой оболочки с точки зрения долговременного термостатирования построена зависимость коэффициента теплопередачи от толщины стенки.

\section{Результаты и обсуждение}

На основе приведенных алгоритмов расчетов нами были определены характеристики и конструкция основных систем транспортного корабля.

В качестве основного вида несущего газа выбран горячий воздух, дополнительного гелий. Преимуществом воздуха является финансовая и территориальная доступность, что обусловливает возможность складывания оболочки для хранения. Гелий позволяет при необходимости увеличивать массу максимальной полезной нагрузки, что делает транспортную систему более универсальной.

По результатам проектного расчета нами были определены характеристики дирижабля, представленные в таблице.

По результатам расчета тангенциальной системы обдува оболочки были получены следующие параметры: дистанция срыва потока и количество щелей $x_{\text {кр }}=0,33$ м и $N=798$, ширина щели $\delta=1$ мм, эквивалентный диаметр входного импеллера и пло- 
щадь, соответствующая этому импеллеру, $D_{\text {им }}=14,283$ м и $S_{\text {им }}=160 \mathrm{м}^{2}$.

Результаты проектного расчета

\begin{tabular}{|c|l|c|}
\hline $\begin{array}{c}\text { № } \\
\text { п/п }\end{array}$ & \multicolumn{1}{|c|}{ Параметр } & Значение \\
\hline 1 & Объем, м & \\
\hline 2 & Масса, кг & 815370 \\
\hline 3 & Крейсерская скорость, км/ч & 394920 \\
\hline 4 & Оптимальная высота полета, км & 6,28 \\
\hline 5 & Длина×ширина×высота, м & $225,2 \times 120,5 \times 76,6$ \\
\hline
\end{tabular}

Впоследствии площадь нагнетания была образована группой из десяти импеллеров диаметром 4,3 м, образующих основную двигательную установку. Параметр протяженности щели $k$ примем равным 20. Протяженность щели, с одной стороны, задает направление потока, но с другой - повышает трение.

По результатам оценочного расчета на прочность элементов спиралевидных рукавов и оценки влияния на жесткость применения двух слоев спиралей было выявлено, что жесткость, по сравнению с одним слоем трубок, увеличивается. Расчетный радиус рукава спирали равен 0,975 м, радиус трубки спирали - 0,65 м.

Определены параметры тросов системы с $K_{3}=1,2$. Выбранный вариант распределения тросов: 20 тросов на один виток баллонета и 71 место крепления в продольном направлении $(3,17$ м между закреплениями в продольном направлении).

Результаты теоретического расчета теплоизоляционных свойств функциональной оболочки были подтверждены расчетом в ПК SolidWorks Simulation. Для выбора оптимального варианта размеров и конструкции стенки газовой оболочки с точки зрения долговременного термостатирования нами была построена зависимость коэффициента теплопередачи от толщины стенки $k_{\text {ст }}\left(\delta_{\text {ст }}\right)$. Наиболее оптимальным вариантом является случай с двумя спиральными рукавами, при котором коэффициент теплопередачи и толщина стенки $k_{\text {ст }}=0,784$ и $\delta_{\text {ст }}=0,97$ м.

Таким образом, нами определены характеристики и конструкция основных систем транспортного дирижабля. Общий вид дирижабля представлен на рисунке.

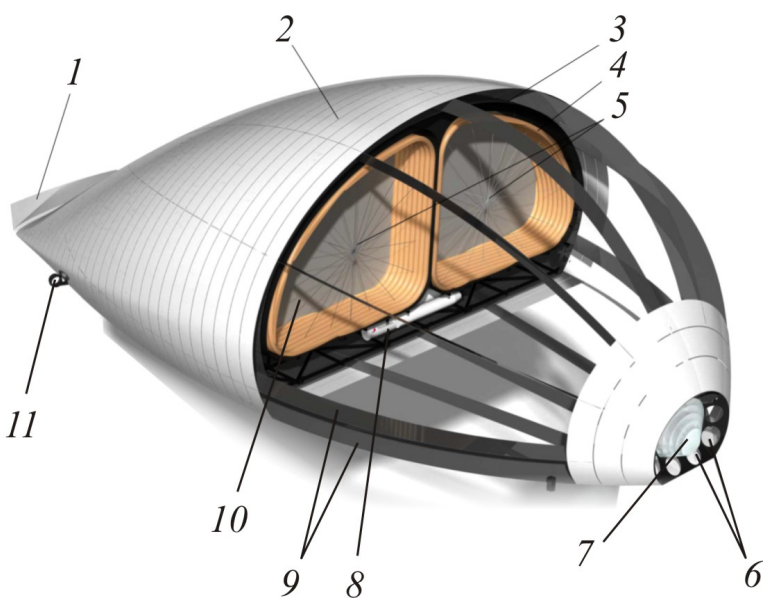

Рис. Общий вид дирижабля: 1 - стабилизатор; 2 - аэродинамическая оболочка; 3 - функциональная оболочка; 4 - спиральный каркас газовой оболочки; 5 - тросы каркаса газовой оболочки; 6 - основная двигательная установка; 7 - рубка управления; 8 - полезный груз; 9 - стрингер подкрепляющей конструкции аэродиномической оболочки; 10 - перегородка между секциями газовой оболочки; 11 - дополнительная двигательная установка

\section{Выводы}

Дирижабельный транспорт является видом транспорта, ранее не применявшимся для доставки грузов РКТ. В нашем проекте мы предлагаем применить инновационные подходы в конструкции силовой и аэродинамической схем: квазижесткая силовая схема аппарата на основе спиралевидных рукавов, наддуваемых избыточным давлением, и система тангенциального обдува оболочки дирижабля.

Итоги нашей работы следующие:

1. Проанализированы актуальные проблемы ракетно-космической отрасли.

2. Определены точные эксплуатационные условия на маршруте Москва - Самара космодром «Восточный».

3. Определен облик дирижабля и основные аэродинамические характеристики полученной формы.

4. Исходя из основных факторов, влияющих на конструкцию, определен облик функциональной оболочки, системы тангенциального обдува, несущей фермы, грузового отсека и системы швартовки, а также рассчитаны основные конструкторские параметры данных систем.

5. Проработана конструкция вышеупомянутых систем. 


\section{Библиографический список}

1. Основные положения основ государственной политики Российской Федерации в области космической деятельности на период до 2030 года и дальнейшую перспективу, утвержденные Президентом Российской Федерации от 19 апреля 2013 г. [Электронный ресурс]. - № Пр-906. - URL: http://www. roscosmos.ru/media/files/docs/3/osnovi_do_2030.doc (дата обращения: 16.10.2020).

2. Zolin A., Didkovsky A. Development of the transport airship for cargo delivery to the Vostochny Cosmodrome // AIP Conf. Proceed., Moscow, Russia, 28 January - 1 February 2019. - Moscow: AIP Publishing LLC, 2019. - Vol. 2171, no. 1. - P. 120004.

3. Kirilin A. Do new generation airships change a paradigm in transport logistics? // Proceed. of the 10th Int. Airship Convention \& Exhibition, Friedrichshafen, Germany, 16-18 April, 2015. - Friedrichshafen, Germany, 2015. - Paper 17. - P. 1-14.

4. Кирилин А.Н. Создание воздушной транспортной системы на основе дирижаблей нового поколения для освоения труднодоступных регионов России // Ежемесячный национальный авиационный журнал «Крылья Родины». - 2019. - № 1-2. - С. 38-39.

5. Prentice B.E., Knotts R. Cargo airships: international competition // Journal of Transportation Technologies. - 2014. - No. 4 (3). - P. 187-195.

6. Кирилин А.Н. Дирижабли. - М.: Изд-во МАИ-ПРИНТ, 2013. - 415 с.

7. Грумондз В.Т., Семенчиков Н.В., Яковлевский О.В. Аэромеханика дирижабля. - М.: Наука, 2017. -424 c.

8. Azouz N., Bestaoui Y., Lemaitre O. (2002, November). Dynamic analysis of airships with small deformations // Proceed. of the Third Int. Workshop on Robot Motion and Control. RoMoCo'02, Bukowy Dworek, Poland, 11 November 2002. - Bukowy Dworek, Poland, IEEE, 2002. - P. 209-215.

9. Bolkcom C. Potential military use of airships and aerostats // Library of Congr. Washington DC Cong. Res. Serv. - 2006. - RS21886. -6 p.

10. Colozza A. Airships for planetary exploration. - 2004. - NASA/CR-2004-213345. - 10 p.

11. Dick H., Robinson D. The golden age of the great passenger airships: Graf Zeppelin and Hindenburg. Washington, DC: Smithsonian Institution Press, 1991. - 226 p.

12. Thermal calculation of airship hull protection from snow / A.N. Kirilin, A.A. Boldyreva, S.F. Timushev, A.V. Tsipenko // Global Journal of Pure and Applied Mathematics. - 2016. - Vol. 12, no 1. - P. 602-615.

13. Бутылин И.Д., Фомин В.М., Щуров А.А. Управление отрывом пограничного слоя // Ученые записки ЦАГИ. - 1991. - Т. ХХІІ, № 2. - С. 6.

14. Овчинников В.А. Устойчивость оптимально управляемого ламинарного пограничного слоя на плоской пластине // Математическое моделирование и краевые задачи. - 2005. - Ч. 2. - С. $194-197$.

15. Виленский Я.Г. Управление пограничным слоем крыла // Труды ЦАГИ. - 1945. - № 565. - С. 48.

\section{References}

1. Main provisions of the state policy of the Russian Federation in the field of space activities for the period up to 2030 and beyond, approved by the President of the Russian Federation No. PR-906 of April 19, 2013. URL: http:/www. roscosmos.ru/media/files/docs/3/osnovi_do_2030.doc (Date of treatment: 10/16/2020).

2. Zolin A., Didkovsky A. Development of the transport airship for cargo delivery to the Vostochny Cosmodrome. AIP Conference Proceedings, Moscow, Russia, 28 January - 1 February 2019. AIP Publishing LLC, 2019, vol. 2171, no. 1, p. 120004.

3. Kirilin A. Do new generation airships change a paradigm in transport logistics? Paper 17, Proceedings of the 10th International Airship Convention\& Exhibition. Friedrichshafen, Germany, April, 16-18, 2015, P. 1-14.

4. Kirilin A.N. Sozdaniye vozdushnoy transportnoy sistemy na osnove dirizhabley novogo pokoleniya dlya osvoyeniya trudnodostupnykh regionov Rossii [Creation of an air transport system based on a new generation of airships for the development of remote regions of Russia]. Monthly national aviation magazine "Krylya Rodiny". No. 1-2, 2019, pp. 38-39.

5. Prentice B.E., \& Knotts, R. (2014). Cargo airships: international competition. Journal of Transportation Technologies, 2014, no. 4 (3), pp. 187-195.

6. Kirilin A.N. Dirizhabli [Airships]. MAI-PRINT publishing house, 2013, 415 p.

7. Grumondz V.T., Semenchikov N.V., Yakovlevskiy O.V. Aeromekhanika dirizhablya [Aeromechanics of the airship]. Moscow: Nauka, 2017, 424 p. 
8. Azouz N., Bestaoui Y., \& Lemaitre O. Dynamic analysis of airships with small deformations. In Proceedings of the Third International Workshop on Robot Motion and Control, 2002. RoMoCo'02, Bukowy Dworek, Poland, 11 November 2002, IEEE, pp. 209-215.

9. Bolkcom C. Potential military use of airships and aerostats. Library of Congress Washington DC Congressional Research Service, 2006, RS21886, 6 p.

10. Colozza A. Airships for Planetary Exploration. 2004, NASA/CR-2004-213345, 10 p.

11. Dick H., \& Robinson D. The golden age of the great passenger airships: Graf Zeppelin and Hindenburg. DC - Smithsonian Institution Press, 1991, 226 p.

12. Kirilin A.N., Boldyreva A.A., Timushev S.F., Tsipenko A.V. Thermal calculation of airship hull protection from snow. Global Journal of Pure and Applied Mathematics. ISSN 0973-1768, 2016, vol. 12, no. 1, pp. 602-615.

13. Butylin I.D., Fomin V.M., Shchurov A.A. Upravleniye otryvom pogranichnogo sloya [Managing the separation of the boundary layer]. Uchenyye zapiski TsAGI, 1991, vol. XXII, no. 2, 6 p.

14. Ovchinnikov V.A. Ustoychivost optimalno upravlyayemogo laminarnogo pogranichnogo sloya na ploskoy plastine [Stability of an optimally controlled laminar boundary layer on a flat plate]. Mathematical Modeling and Boundary Value Problems, 2005, part 2, pp. 194-197.

15. Ya.G. Vilenskiy. Upravleniye pogranichnym sloyem kryla [Control of the wing boundary layer]. Trudy TsAGI, 1945, No. 565, 48 p. Moscow: Publishing house of the Bureau of new technology of the NKAP.

\section{Об авторах}

Дидковский Аркадий Александрович (Москва, Россия) - студент кафедры «Стартовые ракетные комплексы» ФБОУ ВО МГТУ им. Н.Э. Баумана (105005, Москва, ул. 2-я Бауманская, д. 5, e-mail: DidkovskiyAA@yandex.ru).

Мухина Екатерина Дмитриевна (Москва, Россия) - студентка кафедры «Стартовые ракетные комплексы» ФБОУ ВО МГТУ им. Н.Э. Баумана (105005, Москва, ул. 2-я Бауманская, д. 5).

Станишевский Глеб Юрьевич (Москва, Россия) - студент кафедры «Стартовые ракетные комплексы» ФБОУ ВО МГТУ им. Н.Э. Баумана (105005, Москва, ул. 2-я Бауманская, д. 5).

\section{About the authors}

Arkadiy A. Didkovskiy (Moscow, Russian Federation) - Student of Launch Rocket Systems Department, Moscow State Technical University named by N.E. Bauman (5, 2nd Bauman st., Moscow, 105005, Russian Federation, e-mail: DidkovskiyAA@yandex.ru).

Ekaterina D. Mukhina (Moscow, Russian Federation) - Student of Launch Rocket Systems Department, Moscow State Technical University named by N.E. Bauman (5, 2nd Bauman st., Moscow, 105005, Russian Federation).

Gleb Yu. Stanishevskiy (Moscow, Russian Federation) - Student of Launch Rocket Systems Department, Moscow State Technical University named by N.E. Bauman (5, 2nd Bauman st., Moscow, 105005, Russian Federation).

Получено 16.10.2020 